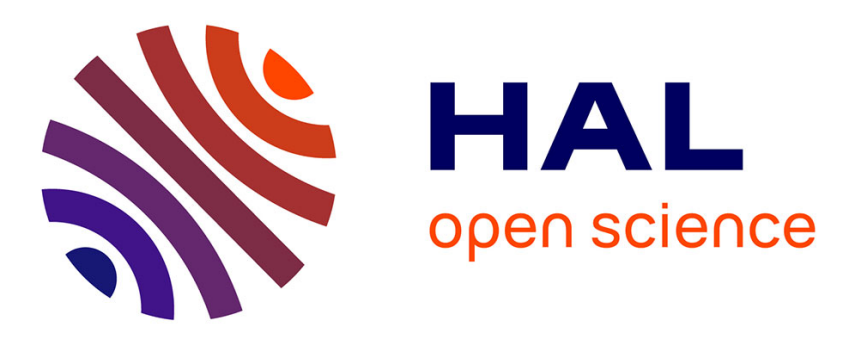

\title{
Atomic diffraction under oblique incidence: An analytical expression
}

Maxime Debiossac, Philippe Roncin

\section{To cite this version:}

Maxime Debiossac, Philippe Roncin. Atomic diffraction under oblique incidence: An analytical expression. Physical Review A : Atomic, molecular, and optical physics [1990-2015], 2014, 90 (5), 10.1103/PhysRevA.90.054701 . hal-01417306

\section{HAL Id: hal-01417306 \\ https://hal.science/hal-01417306}

Submitted on 12 Oct 2021

HAL is a multi-disciplinary open access archive for the deposit and dissemination of scientific research documents, whether they are published or not. The documents may come from teaching and research institutions in France or abroad, or from public or private research centers.
L'archive ouverte pluridisciplinaire HAL, est destinée au dépôt et à la diffusion de documents scientifiques de niveau recherche, publiés ou non, émanant des établissements d'enseignement et de recherche français ou étrangers, des laboratoires publics ou privés. 


\title{
Atomic diffraction under oblique incidence: An analytical expression
}

\author{
Maxime Debiossac and Philippe Roncin \\ Institut des Sciences Moléculaires d'Orsay (ISMO), CNRS-Univ Paris-Sud, UMR 8214 Bât. 351, Univ Paris-Sud, F-91405 Orsay, France
}

(Received 29 April 2014; revised manuscript received 25 September 2014; published 13 November 2014)

\begin{abstract}
The semiclassical perturbation method developed by Henkel et al. [J. Phys. II 4, 1955 (1994)] to model cold-atom diffraction by optical standing waves, is applied to the diffraction of fast atoms on crystal surfaces at grazing incidence (GIFAD or FAD). We first show that the interaction time and interaction length embedded in the obliquity factor is well suited to explain the transition from three-dimensional to two-dimensional (2D) diffraction. The situation of a slightly misaligned primary beam, corresponding to oblique incidence in the effective 2D system, is addressed pointing out discrepancies such as the absence of net deflection of the atomic beam. Guided by time-reversal considerations, we propose an arbitrarily symmetrized form significantly improving the agreement with experimental data recorded in oblique incidence.
\end{abstract}

DOI: 10.1103/PhysRevA.90.054701

PACS number(s): $34.35 .+\mathrm{a}, 68.49 . \mathrm{Bc}, 34.50 . \mathrm{Cx}$

Since the development of quantum atom optics, in particular interferometry with cold atoms and molecules [1,2], wave matter diffraction phenomena have been observed on light gratings, in transmission [3], and reflection [4]. In this context, the development of a simple analytical formula to model both situations as a thin phase grating by Henkel et al. [5] has received considerable attention. In a different context, diffraction of thermal atoms and molecules at crystal surfaces, first observed by Estermann and Stern in 1930 [6], has developed as a specific surface science technique able to reveal the corrugation amplitude of the surface electronic density in the sub- $\AA$ range. More recently, the diffraction of fast, $\mathrm{keV}$, atoms off crystalline insulator, metal, or semiconductor surfaces at grazing incidence (GIFAD) has been demonstrated both under static [7-10] and conventional thin-film growth conditions [11]. Theoretically, extensive semiclassical techniques based on the eikonal approximation with quantum treatment of the rainbow [12,13], sophisticated wave-packet approaches [9,14,15], and close coupling calculations [16] have been developed reaching excellent accuracy but are computationally too heavy to be used in real time during experiments. At normal incidence, analytical formula already exist that provided few picometer sensitivity on the corrugation amplitude of simple lattice cell [15] or even super structure such as the moire pattern of graphene [17], but no equation is available that can reproduce even qualitatively diffraction intensity when the beam is misaligned by a fraction of a degree from the crystal axis.

\section{ATOMIC DIFFRACTION BY A THIN PHASE GRATING}

In Ref. [5] Henkel et al. study the reflection of atoms on the evanescent field created above a dielectric by internal reflection of a laser standing wave. We briefly recall their results while renaming variables according to the context of atom surface scattering. Only in plane diffraction, i.e., the 2D problem is treated explicitly and the approximation starts from the unperturbed atom trajectory corresponding to specular reflection on an planar exponential atom-surface potential $V_{0}(z)=V_{p} \exp \left(-z / R_{c}\right)$. In this simple expression $V_{p}$ is a pre-exponential factor, which has no importance here, while $R_{c}$ is the range of the potential defining the characteristic interaction time with the surface $\tau_{\text {int }}=R_{c} / v_{i z}$, with $v_{i z}$ the velocity component toward the surface. The classical trajectory is analytic [18] allowing semiclassical wave functions to be derived. The action integral $S(x)$ describing the momentum transfer is expanded as a power series $S(x)=S_{0}+\varepsilon S_{1}(x)+$ $\varepsilon^{2} S_{2}(x)+O\left(\varepsilon^{3}\right)$ of the corrugated part of the potential taken here as a perturbation; $V(x, z)=V_{p}(z) *\left[1+\varepsilon \cos \left(2 \pi x / a_{x}\right)\right]$ $a_{x}$ being the lattice parameter and $G_{x}=2 \pi / a_{x}$ the reciprocal lattice vector. As a result, they derive the diffracted intensities $I_{n}$ for any oblique incidence:

$$
I_{n}=\frac{k_{f z}}{k_{i z}} J_{n}^{2}\left[2 k_{i z} \varepsilon R_{c} \chi\left(2 R_{c} G_{x} \frac{k_{i x}}{k_{i z}}\right)\right],
$$

where $J_{n}$ is the Bessel function of the first kind of order $n, k_{f z} / k_{i z}$ a geometrical factor due to normalization of the wave function, and $\chi\left(2 R_{c} G_{x} \frac{k_{i x}}{k_{i z}}\right)$ with $\chi(u)=u / \sinh (u)$ is the obliquity factor associated with the incidence angle $\theta_{\text {in }}$ defined by $\tan \left(\theta_{\text {in }}\right)=k_{i x} / k_{i z}$.

At normal incidence $\left(\theta_{\text {in }}=0\right)$, the obliquity factor is equal to 1 and can be forgotten. For weak contrast $\varepsilon \ll 1$ the classical turning point $Z_{t}$ is shown to have a cosine dependence $Z_{t}(x)=c s t+\varepsilon R_{c} \cos \left(G_{x} x\right)$. Introducing the full (top to bottom) corrugation amplitude $h_{c}=2 \varepsilon R_{c}$ the diffracted intensity becomes $I_{n}=\frac{k_{i x}}{k_{i z}} J_{n}^{2}\left(2 k_{i z} h_{c}\right)$ similar to [19] and to the corrugated hardwall model where a Fresnel-Huygens ray tracing model retrieves the intensities diffracted from a corrugated mirror. At this normal incidence, the interest of Eq. (1) appears therefore limited because the newly introduced $R_{c}$ parameter is entangled with the corrugation amplitude $h_{c}$ without adding new prediction.

For incidence angle departing from normal, the obliquity factor is less than one and corresponds a reduced coupling leading to a reduced population of high diffraction orders. The argument $u$ describes the number of lattice sites span within the range $R_{c}$. More precisely the interaction length $L_{x}$ simply defined as $L_{x}=v_{i x} \tau_{\text {int }}$ allows the argument $u$ to be be rewritten as $u=4 \pi L_{x} / a_{x}=2 G_{x} L_{x}$ proportional to the number $L_{x} / a_{x}$ of lattice sites within the range during the interaction length. Since the interaction oscillates with a spatial period $a_{x}$, the obliquity factor describes the progressive cancellation of all the contributions of successive lattice sites as the angle of incidence becomes more and more grazing. This was also discovered numerically by Farias et al. [20,21] when 
reducing down to $10 \mathrm{deg}$ the angle of incidence of thermal hydrogen with respect to the surface plane $\left(\theta_{\text {in }}=80 \mathrm{deg}\right.$ to the normal). Note also that the above defined trajectory length was shown to be a very useful concept to derive a fundamental parameter such as the energy and momentum exchange with the lattice $[12,22,23]$ or Auger neutralization rates at insulator surface [24] or metals [25] in the context of grazing incidence.

\section{GRAZING INCIDENCE FAST ATOM DIFFRACTION (GIFAD)}

GIFAD setups have been detailed in numerous papers [7-9]. It consists of a primary $\mathrm{He}^{+}$ion beam extracted at the desired final energy from a commercial ion source. The $\mathrm{He}^{+}$ions are converted to a fast neutral $\mathrm{He}^{0}$ atom via resonant charge exchange inside an effusive gas cell. The $\mathrm{He}^{0}$ beam is then collimated before entering the chamber hosting the target surface. Figure 1 defines the two relative angles of the primary neutral beam with respect to a selected low index direction of the crystal surface. The diffracted beams are collected $\approx 1 \mathrm{~m}$ downstream onto a position sensitive detector. In practice, both $\Gamma$ and $\phi$ angles hardly exceed $1 \mathrm{deg}$. It should be noted that the geometry is exactly the one used in RHEED setup widely used in the molecular beam epitaxy chamber to monitor thin film growth where GIFAD can therefore be an alternative technique [11].

Starting from the first GIFAD observations [7-9] it was realized that diffracted intensities corresponding to the exchange of reciprocal lattice vector $G_{x}$ (Fig. 1) are not observed. In terms of energy, and assuming $G_{x} \sim G_{y}$, such an exchange along $x$ costs $k_{i} / G$ times more than when the exchanged vector is perpendicular to the initial momentum (i.e., along $y$ ). Interestingly the other argument raised [9,20,21], in addition to

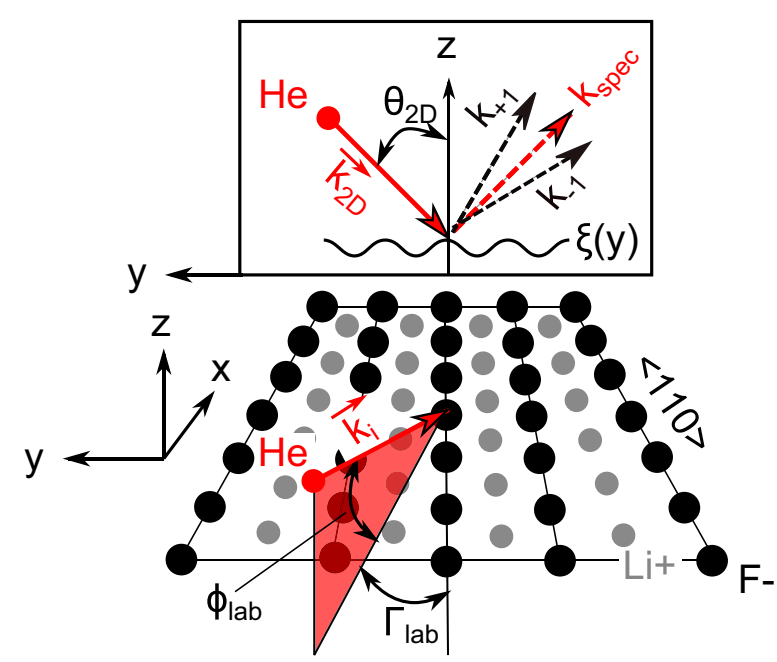

FIG. 1. (Color online) Schematic of the scattering geometry. A fast $\mathrm{He}$ atom is incident with a total wave vector $\vec{k}_{i}$ close to the $\langle 110\rangle$ direction of $\mathrm{LiF}(100)$ surface with polar incidence angle $\phi_{\text {lab }}$ and misalignment angle $\Gamma_{\text {lab }}$. This 3D scattering problem can be reduced to a $2 \mathrm{D}$ problem of the He atom probing, with a wave vector $\vec{k}_{i, 2 \mathrm{D}}=$ $\left(k_{i y}, k_{i z}\right)$, the corrugation function $\xi(y)$ under oblique incidence $\theta_{2 \mathrm{D}}=$ $\arctan \left(k_{i y} / k_{i z}\right)$. The diffracted beams are depicted with dashed vectors with the specular one in red. the energy increase, is that the associated coupling decreases at the grazing angle for geometric reasons and this is exactly what is described in the obliquity factor. For the geometry used in Sec. III, the numerical value is vanishingly small forcing specular reflection in the scattering plane. If any, diffraction has be out of plane, as actually observed (from there on $G_{y}$ will be used instead of $G_{x}$ ). As a result the movement along the $x$ direction can be reduced to a pure translation and eliminated. Of course, doing so one has to consider the effective interaction potential defined as the average of the 3D $V(x, y, z)$ potential along the $x$ direction [9]. The 3D egg boxlike turns into a corrugated iron plate, both having the same exponential range $R_{c}$. If we define the effective interaction parameters as those in the $(y, z)$ plane (the detector plane) one gets $\vec{k}_{2 \mathrm{D}}=$ $k_{i}\left(\sin \left(\phi_{\mathrm{lab}}\right), \sin \left(\Gamma_{\mathrm{lab}}\right)\right)$ and $\tan \left(\theta_{2 \mathrm{D}}\right)=\tan \left(\Gamma_{\mathrm{lab}}\right) / \tan \left(\phi_{\mathrm{lab}}\right)$. The most important result being that the effective interaction energy can be continuously adjusted between a few meV to few eV allowing the exploration of the electron density on a range of distances to the atomic plane comparatively wider than HAS. The progressive transition from $3 \mathrm{D}$ to $2 \mathrm{D}$ diffraction have been studied in detail with wave packet technique [26,27] and partially observed [28] using a surface with comparatively large super lattice (large a involving many atoms per unit cell) and rather oblique incidence.

For grazing incidence and small misalignment angles, the effective $2 \mathrm{D}$ oblique incidence angle can be written as $\tan \left(\theta_{2 \mathrm{D}}\right) \approx \Gamma_{\text {lab }} / \phi_{\text {lab }}$ so that for $\phi_{\text {lab }}=1 \mathrm{deg}$, a $1 \mathrm{deg}$ misalignment angle yields a 45 deg effective oblique incidence. This outlines that quasinormal effective incidence requires that $\Gamma_{\text {lab }}$ is orders of magnitude smaller than $\phi_{\text {lab }}$ which is extremely demanding in terms of mechanical accuracy, particularly when thermal treatment is required. This explains that many GIFAD images are recorded with a slightly oblique incidence.

The systematic experimental investigation of the diffracted intensity as a function of the misalignment angle $\Gamma$ was first reported on $\mathrm{LiF}$ surface [29] followed by a detail analysis and associated calculation with close coupling equations [14]. Note that during such a $\Gamma$ scan the modulus of the effective wave vector $k_{2 \mathrm{D}}$ is not constant (only $k_{i z}$ is constant). Both studies indicate that the diffracted profile becomes strongly asymmetric $\left(I_{+n} \neq I_{-n}\right)$ resulting in a net deflection of the diffracted beam taken as a whole, i.e., the average deflection angle $\left\langle\Gamma_{\text {out }}\right\rangle-\Gamma_{\text {in }} \propto \Sigma_{n} n I_{n}$ is not zero and shows a characteristic behavior with a linear dependence around $\Gamma=0$, allowing automatic alignment of the crystal [14]. The prediction of Eq. (1) would here be a flat horizontal line because it predicts a symmetric population of the diffraction orders $\left(I_{+n}=I_{-n}\right)$.

In their work on beam deflection [14], the authors use time-reversal symmetry [30] to link the data corresponding to oblique incidence to those recorded under normal incidence taking into account the modified energy in the effective $(y, z)$ plane. This symmetry is not present in Eq. (1), it does not predict the same scattering probability for $0 \rightarrow n$ and $n \rightarrow 0$. This shortcoming probably derives from the fact that the obliquity factor is evaluated only along the specular trajectory. One could try to imagine half trajectories where the action integral would be evaluated separately on the way in and out. This being beyond our forces we have tried to force the time symmetry by modifying arbitrarily formula (1) so 
that it gives the same result if one exchanges the labels " $i$ " and " $f$ " [31]. In practice the initial momenta $k_{i y}$ and $k_{i z}$ are replaced by their average between initial and final values $k_{i z} \rightarrow \bar{k}_{z}=\left(k_{i z}+k_{f z}\right) / 2$ and $k_{i y} \rightarrow \bar{k}_{y}=\left(k_{i y}+k_{f y}\right) / 2$, with $k_{f y}=k_{i y}+n G_{y}$ and $k_{f z}=\sqrt{k_{i z}^{2}-2 n G_{y} k_{i y}-n^{2} G_{y}^{2}}$, Eq. (1) becomes

$$
I_{n}=\frac{k_{f z}}{k_{i z}} J_{n}^{2}\left[2 \bar{k}_{z} h_{c} \chi\left(2 R_{c} G_{y} \frac{\bar{k}_{y}}{\bar{k}_{z}}\right)\right] .
$$

It should be noted that the argument inside the Bessel function now depends on the diffraction order.

\section{RESULTS AND DISCUSSION}

We now compare the prediction of Eq. (2) in the case of fast atom diffraction on a $\mathrm{LiF}(100)$ surface when the incident beam is aligned close to the $\langle 110\rangle$ direction. This surface and direction has been extensively studied and quasisinusoidal equipotential curves have been reported in the sub eV effective energy range with a corrugation amplitude small enough to prevent multiple scattering. The parameters $R_{c}=1.18 \AA$ and $h_{c}=0.325 \AA$ have been fitted to reproduce the DFT potential of LiF surface given in [32].

In Fig. 2 we show the diffraction chart for semiclassical calculations in oblique incidence obtained from Eq. (1) [Fig. 2(d)] and from Eq. (2) [Fig. 2(b)] together with the experimental data and a quantum calculation based on the close coupling formalism [14] for ${ }^{4} \mathrm{He}$ at $E_{i}=460 \mathrm{eV}$ incident at $\phi_{\text {in }}=0.93^{\circ}$ aligned close to the $\langle 110\rangle$ direction of $\operatorname{LiF}(100)$. For the specular order, both semiclassical models give the same diffracted intensity but for higher diffraction orders (i.e., for larger oblique incidence angle $\theta_{i}$ ) the diffracted intensity is no longer symmetric with respect to $k_{i y}=0$ in the case of Eq. (2). In order to emphasize the link with classical mechanics we have calculated the extrema of the momentum transfer from the classical equation of motion. The result is shown as dashed lines in Fig. 2(b) and the position of the rainbow angle where the momentum transfer is maximum is highlighted $\left(k_{i y \text {,rainbow }} \approx 4 G_{y}\right)$. At this value of misalignment, Fig. 3 compares, in a more quantitative way, diffraction intensities [experiment, quantum, and semiclassical from Eq. (2)].

We see from Figs. 2 and 3 that the semiclassical approach proposed here reproduces surprisingly well the experimental data which is very encouraging for practical applications. Of course a deeper analysis of this ad hoc modification is certainly needed on the theoretical point of view. For instance, the geometrical prefactor $k_{f z} / k_{i z}$ is not symmetric either, other symmetrized forms are also possible and may be easier to justify, etc. More pragmatic, possible extensions beyond pure sinusoidal corrugation function can also be investigated. Intuitively, there is a reasonable hope that the actual constraint is that the corrugation function integrates to zero over a lattice unit, i.e., for symmetric cells. Another very useful extension would be to link directly this model to the 2D row model developed for grazing incidences [33], where the corrugation amplitude may depend on the effective energy. Concerning the limitations, these should the same as exposed in [5] knowing that the intensities are supposed to be better described than the associated phases. The limit for the semiclassical approach

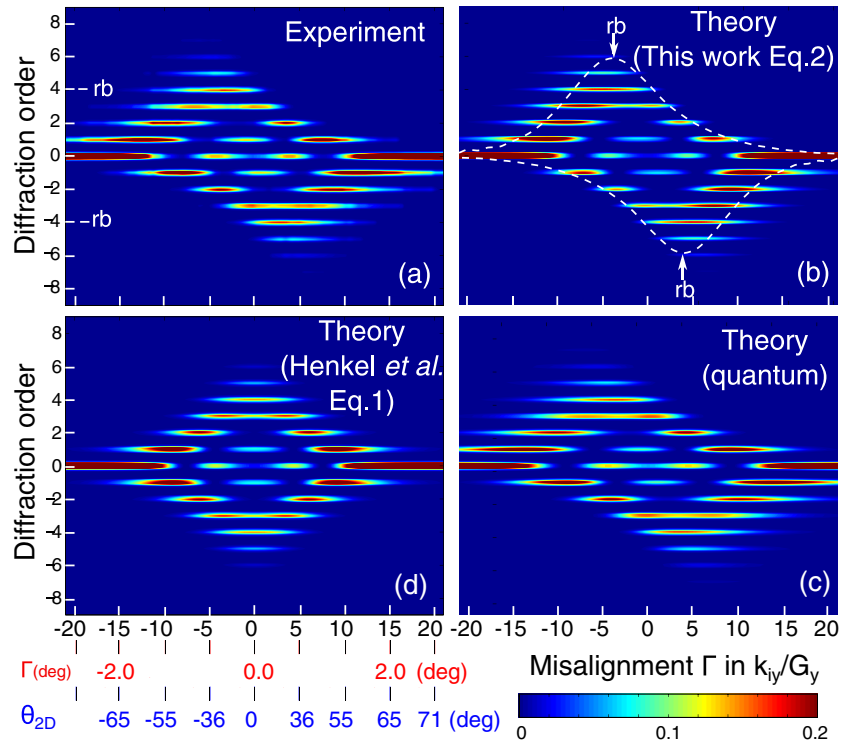

FIG. 2. (Color online) Diffraction charts for ${ }^{4} \mathrm{He}$ with $E_{i}=$ $460 \mathrm{eV}$ and $\phi_{\text {lab }}=0.93^{\circ}$ incident on a LiF surface close to the $\langle 110\rangle$ direction, i.e., $k_{i}=942 \AA^{-1}$ and $k_{i z}=15.3 \AA^{-1}, G_{y}$ is $2.2 \AA^{-1}$ corresponding to a deflection angle of $0.134 \mathrm{deg}=\sin ^{-1}\left(G_{y} / k_{y}\right)$. The interaction parameters are $R_{c}=1.18 \AA$ and $h_{c}=0.325 \AA$. (a) Experimental data [14]. (b) Semiclassical calculations using Eq. (2). (c) "Exact" quantum calculation [14]. (d) Semiclassical calculations using Eq. (1). Note that both horizontal and vertical scale have the same units $\left(G_{y}\right)$ relevant for diffraction. In addition, the misalignment angle $\Gamma_{\text {lab }}$ is reported also in degrees in the laboratory frame (red) as well as the corresponding obliquity angle $\theta_{2 \mathrm{D}}=\tan \left(\Gamma_{\mathrm{lab}}\right) / \tan \left(\phi_{\text {lab }}\right)$ (blue). (a) and (c) have been adapted from [14]. The dashed line in (b) correspond to the maximum transfer of momentum obtained from classical calculations. The rainbow angle of $k_{i y} \approx 4 G_{y}$ used for quantitative comparison in Fig. 3 is marked by the arrows labeled "rb."

$\lambda_{2 \mathrm{D}} \lesssim R_{c}$ is easily fulfilled for helium particles with $E_{2 \mathrm{D}}$ above $1 \mathrm{meV}$ because the range $R_{c}$ is always on the order of magnitude of few bohr radius (0.523 $\AA$ ) [34]. This is because $R_{c}$ derives from fundamental atom-atom interaction potential

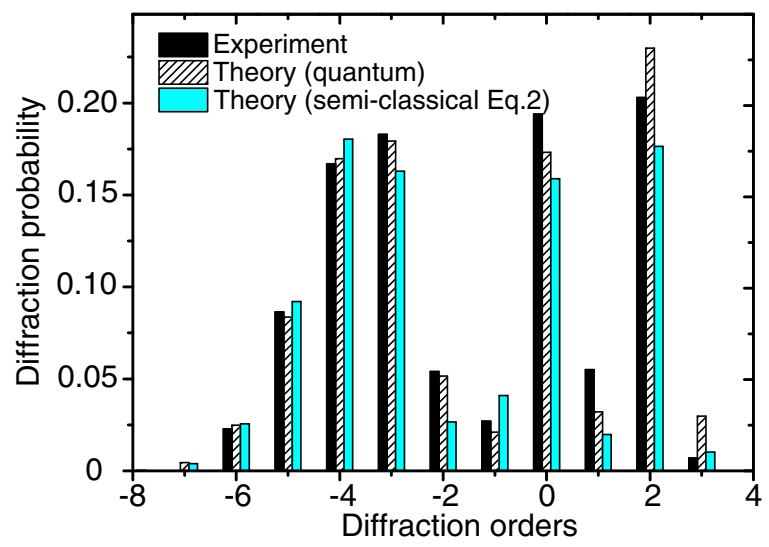

FIG. 3. (Color online) Diffraction probabilities extracted from Fig. 2 for $k_{i y}=4 G_{y}$ (rainbow position) for experiment (black pattern), quantum theory (dashed pattern), and semiclassical theory from Eq. (2) (blue pattern). 
which, in the asymptotic region probed here, is governed by the simple overlap of the ground state wave function scaling as $I_{p}^{1 / 2}$ with $I_{p}$ the ionization potential and $I_{p}$ does not vary significantly within the periodic table. As far as simple lattice cells are concerned, the lattice parameter $a$ is on the order of 10 atomic units so that the condition of weak corrugation $h_{c} \ll a_{y}$ is not too restrictive if no surface reconstruction involving several atomic layers is present.

\section{SUMMARY AND CONCLUSION}

We have shown that the perturbation method developed for the diffraction on light grating [5] is well suited to account for reflection on surfaces at normal incidence. We have suggested that the obliquity factor has a strong physical support explaining the collapse of in plane diffraction as the angle of incidence becomes grazing. However, as is, this obliquity factor seems to violate the time-reversal symmetry and fails to account for the scattering asymmetry. By imposing a more symmetric form we have shown that the agreement with experiment is greatly improved. Our modified Eq. (2) should have the same physical limitations as the original formula, i.e., it only applies to diffraction in the semiclassical regime and from weakly corrugated potential where multiple scattering events do not occur. Optimistically, the good agreement presented here suggest that $\Gamma$ scans exploring large misalignment could allow experiment measurement of the effective range $R_{c}$ of the interaction potential. If so, the technique would be very useful because, once again, $R_{c}$ is a very important physical parameter governing the interaction length, the number of active sites, the momentum, and energy transfer to the surface. This would provide a direct (self-consistent) link with the angular dependent effective number of sites $N(\phi)$ included in the Debye-Waller factor modified for grazing incidence $[12,23,35]$. This drastic reduction of the original Debye-Waller factor by $e^{-N(\phi)}$ is at the heart of the ability of GIFAD to explore the surface at high temperature $[11,16]$ as required for operation inside a molecular beam epitaxy chamber. This work was motivated by the urgent need for a simple approach withstanding a slight misalignment in the GIFAD diffraction data. Since the result is analytic we believe that, in complement with more rigorous methods, it will prove useful as a fast method to qualitatively benefit from the picometer sensitivity of GIFAD.

\section{ACKNOWLEDGMENTS}

We acknowledge C. Westbrook, J. R. Manson, and P. Atkinson for helpful discussions and corrections in the writing of the manuscript. This work has been funded by the Agence Nationale de la Recherche (Contract ANR-2011EMMA-003-01).
[1] P. R. Berman, Atom Interferometry (Academic, New York, 1997).

[2] T. Juffmann, H. Ulbricht, and M. Arndt, Rep. Prog. Phys. 76, 086402 (2013).

[3] D. W. Keith, M. L. Schattenburg, H. I. Smith, and D. E. Pritchard, Phys. Rev. Lett. 61, 1580 (1988)

[4] J. V. Hajnal and G. I. Opat, Opt. Commun. 71, 119 (1989).

[5] C. Henkel, J.-Y. Courtois, and A. Aspect, J. Phys. II 4, 1955 (1994).

[6] I. Estermann and O. Stern, Z. Phys. 61, 95 (1930).

[7] H. Khemliche, P. Roncin, and P. Rousseau, US Patent No. 8,232,518, April 8, 2009.

[8] A. Schüller, S. Wethekam, and H. Winter, Phys. Rev. Lett. 98, 016103 (2007).

[9] P. Rousseau, H. Khemliche, A. G. Borisov, and P. Roncin, Phys. Rev. Lett. 98, 016104 (2007).

[10] H. Winter and A. Schüller, Prog. Surf. Sci. 86, 169 (2011).

[11] P. Atkinson, M. Eddrief, V. H. Etgens, H. Khemliche, M. Debiossac, A. Momeni, M. Mulier, B. Lalmi, and P. Roncin, Appl. Phys. Lett. 105, 021602 (2014).

[12] J. R. Manson, H. Khemliche, and P. Roncin, Phys. Rev. B 78, 155408 (2008).

[13] M. Gravielle and J. Miraglia [Phys. Rev. A (to be published)], arXiv:1407.5669.

[14] A. Zugarramurdi, M. Debiossac, P. Lunca-Popa, L. S. Alarcón, A. Momeni, H. Khemliche, P. Roncin, and A. G. Borisov, Phys. Rev. A 88, 012904 (2013).

[15] A. Schüller, S. Wethekam, D. Blauth, H. Winter, F. Aigner, N. Simonović, B. Solleder, J. Burgdörfer, and L. Wirtz, Phys. Rev. A 82, 062902 (2010).
[16] M. Debiossac, A. Zugarramurdi, P. Lunca-Popa, A. Momeni, H. Khemliche, A. G. Borisov, and P. Roncin, Phys. Rev. Lett. 112, 023203 (2014).

[17] A. Zugarramurdi, M. Debiossac, P. Lunca-Popa, A. Mayne, A. Momeni, A. G. Borisov, P. Roncin, and H. Khemliche (unpublished).

[18] J. M. Jackson and N. F. Mott, Proc. R. Soc. London Ser. A 137, 703 (1932).

[19] U. Garibaldi, A. Levi, R. Spadacini, and G. Tommei, Surf. Sci. 48, 649 (1975).

[20] D. Farias, C. Diaz, P. Rivière, H. F. Busnengo, P. Nieto, M. F. Somers, G. J. Kroes, A. Salin, and F. Martin, Phys. Rev. Lett. 93, 246104 (2004).

[21] D. Farias, C. Diaz, P. Nieto, A. Salin, and F. Martin, Chem. Phys. Lett. 390, 250 (2004).

[22] J. I. Juaristi, A. Arnau, P. M. Echenique, C. Auth, and H. Winter, Phys. Rev. Lett. 82, 1048 (1999).

[23] P. Rousseau, H. Khemliche, N. Bundaleski, P. Soulisse, A. Momeni, and P. Roncin, J. Phys.: Conf. Ser. 133, 012013 (2008).

[24] P. Rousseau, H. Khemliche, and P. Roncin, Nucl. Instrum. Methods Phys. Res. B 258, 13 (2007).

[25] A. Schüller, G. Adamov, S. Wethekam, K. Maass, A. Mertens, and H. Winter, Phys. Rev. A 69, 050901 (2004).

[26] A. Zugarramurdi and A. G. Borisov, Phys. Rev. A 86, 062903 (2012).

[27] A. Zugarramurdi and A. G. Borisov, Nucl. Instrum. Methods Phys. Res. B 317, 83 (2013).

[28] M. Busch, J. Seifert, E. Meyer, and H. Winter, Phys. Rev. B 86, 241402 (2012). 
[29] J. Seifert, A. Schüller, H. Winter, and K. Gärtner, Nucl. Instrum. Methods Phys. Res. B 269, 1212 (2011).

[30] A. Bohm and M. Loewe, Quantum Mechanics: Foundations and Applications (Springer, Berlin, 1986).

[31] B. Hinch, Surf. Sci. 221, 346 (1989).
[32] A. Schüller, H. Winter, M. S. Gravielle, J. M. Pruneda, and J. E. Miraglia, Phys. Rev. A 80, 062903 (2009).

[33] D. Danailov, K. Gärtner, and A. Caro, Nucl. Instrum. Methods Phys. Res. B 153, 191 (1999).

[34] A. A. Abrahamson, Phys. Rev. 178, 76 (1969).

[35] A. Levi, J. Phys.: Condens. Matter 21, 405004 (2009). 